\title{
Mobility-Non-Exercise Physical Activity (NEPA) is the key to health and longevity
}

\begin{abstract}
ISSN: $2455-5487$
DO :

Received: 05 February, 2019

Accepted: 16 March, 2020

Published: 17 March, 2020
\end{abstract}

\author{
Margaret Arnold ${ }^{1}$ and Joan Vernikos ${ }^{2 *}$ \\ 1PT, CEES, CSPHP, Founder, CEO Inspire Outcomes LLC, Michigan, United States \\ ${ }^{2}$ Life Sciences, NASA and Thirdage Health, Culpeper, VA, United States
}

*Corresponding author: Joan Vernikos, PhD, Life Sciences, NASA and Thirdage Health, Culpeper, VA, United States, E-mail: dr.joan@joanvernikos.com

https://www.peertechz.com

Check for updates

\section{Background}

When asking someone, whatever their age, what they consider most important to their health, the answer invariably involves their ability to maintain physical independence-basic mobility. The highest level of mobility is unlimited movement in any environment they choose. As mobility is lost, the environments in which independent movement is possible, become restricted. As mobility is further compromised, an assistive device may be needed such as a walker or a cane, then with further decline, the person may begin to rely on help for more and more movements, and their independence is replaced by dependence on another for help. The impact of declining mobility on health and well-being, both physical and psychological are substantial and reduced mobility and ability to do Instrumental Activities of Daily Living (IADL) can often be the first sign of declining health Table 1.

Technical advances such as chairs, remote controls, cars and other transportation means, motorized and robotic household and yard appliances have replaced the need for variety of movement throughout the day both in and outside of the home. Work environments and communication technologies have virtually immobilized the office worker by encouraging hours of continuous sedentary behavior, and even the design of office furniture aims to support and spare us the simplest use of our muscles.

Comparisons between physiological effects of microgravity in space, $\left(\mathrm{G} \times 10^{-6}\right)$ bed-rest in healthy volunteers and hospitalized patients ( $\mathrm{G} \times$ through the body gravity) rather than $\mathrm{Gz}$ (Head to toe gravity), and sedentary behavior-uninterrupted sitting-in the elderly and office workers, (decreased use of gravity) have informed our understanding that depriving the human body of

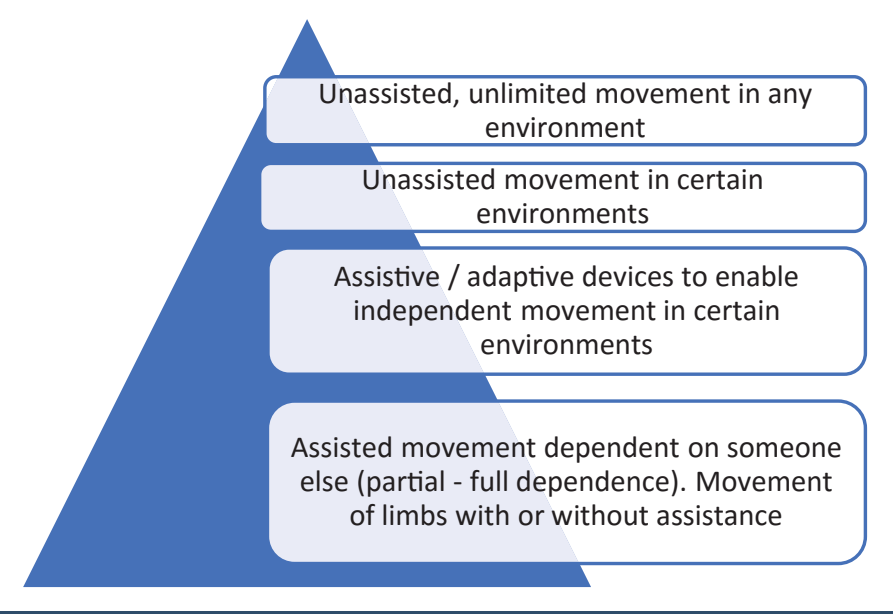

Table 1: Mobility progression and Independence.

gravitational stimulus has many detrimental effects on human health.

Increasing focus on exercise in a gym was initially proposed as a solution, as traditionally, primary exercise health benefits have focused on strength and cardiovascular conditioning. While these health benefits from exercise are important, the type of movement that is being lost in our modern-day lifestyles, that may be even more important to our health is daily general, Non-Exercise Physical Activity (NEPA), which includes interacting with/changing positions relative to gravity [1-5].

Exercise once a day and NEPA throughout the day are not interchangeable. Studies show that once a day exercise in the gym cannot combat the effects of uninterrupted sitting on insulin resistance, blood triglycerides etc. Even in space, 
astronauts exercise vigorously for 2-4hours per day, but this does not combat the physiological impact of gravity deprivation.

Primary care practitioners often ask about exercise, duration and frequency, but do not routinely ask about patients' lifestyle, work-rest environment, social activity or daily habits that may have changed. They may be missing a significant indication of their patients' health status, even before symptoms occur. NEPA should be a standard question addressed at every primary care visit.

Modern day public health epidemics include obesity including more children who are diabetic, metabolic syndrome, type II diabetes, (especially childhood onset), and increasing incidence of cardiovascular diseases as well as many cancers. These conditions are all linked to decreased physical activity. Deprivation of NEPA in modern lifestyles should be a fundamental consideration for preventive and rehabilitative patient care. Increasing NEPA for at risk patients can also provide a safe and easy remedy for a host of modern-day disorders.

\section{The following criteria are important for NEPA:}

a) Activities should be done in vertical, not horizontal plane, i.e. standing.

b) Bouts of activity need to be performed frequently throughout the day, ideally changing position relative to gravity at least once every 30-60minutes.

c) Duration of position does not need to be more than 1 minute. It is the number of changes in position that are more important than the length of time in the new position. The adrenergic response to the stimulus from change of position such as increased heart rate or increased brain blood flow, decays within 30minutes even though you may continue to remain standing.

The human body is designed to move. The most recent edition of ACSM's Guidelines (2018), with current recommendations for exercise testing and practise, raises the issue of the risks of today's increasingly sedentary behaviour to health and mobility. The findings for maintaining optimal physiological health indicate that it is intermittent, vertical alternating movement throughout the day-every 30 to 6ominutes-that is most beneficial [1-5], rather than the total hours sat.

\section{References}

1. Vernikos J (2018) Space and Aging. In. L Young and R Sutton Eds Encyclopedia of Bioastronautics, Springer.

2. Vernikos J, Ludwig DA, Ertl AC, Wade CE, Keil LC, et al. (1996) Effect of standing or walking on physiological changes induced by head down bed rest. Implications for spaceflight. Aviat Space Environ Med 67: 1069-1079. Link: https://bit.ly/2TW5UW8

3. Engelke KA, Doer DF, Convertino VA (1995) A single bout of exhaustive exercise affects integrated baroreflex function after 16 days of head-down tilt. Amer J Physiol 269: R614-R620. Link: https://bit.ly/38XPctA

4. Vernikos J, Schneider VS (2010) Space, Gravity and the Physiology of Aging Parallel or Converging Disciplines? A Mini-Review. Gerontology 56: 157-166. Link: https://bit.ly/2TT8dsZ

5. Vernikos J (2016) Designed to Move. Quill Driver Books, CA.

\section{Discover a bigger Impact and Visibility of your article publication with} Peertechz Publications

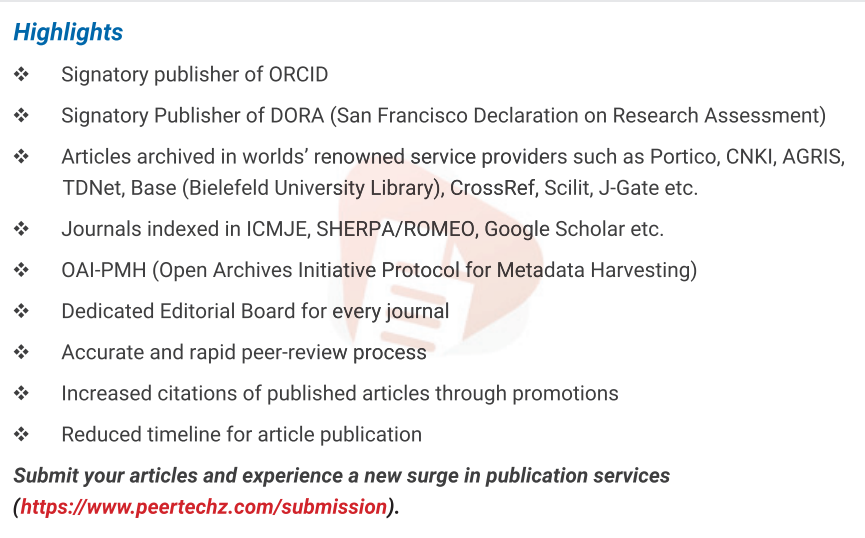

Peertechz journals wishes everlasting success in your every endeavours.

Copyright: ๑ 2020 Arnold M, et al. This is an open-access article distributed under the terms of the Creative Commons Attribution License, which permits unrestricted use, distribution, and reproduction in any medium, provided the original author and source are credited. 